\title{
Route to supersolidity for the extended Bose-Hubbard model
}

\author{
M. Iskin \\ Department of Physics, Koç University, Rumelifeneri Yolu, TR-34450 Sariyer, Istanbul, Turkey
}

(Received 31 January 2011; published 31 May 2011)

\begin{abstract}
We use the Gutzwiller ansatz and analyze the phase diagram of the extended Bose-Hubbard Hamiltonian with on-site $(U)$ and nearest-neighbor $(V)$ repulsions. For $d$-dimensional hypercubic lattices, when $2 d V<U$, it is well known that the ground state alternates between the charge-density-wave (CDW) and Mott insulators, and the supersolid (SS) phase occupies small regions around the CDW insulators. However, when $2 d V>U$, in this Rapid Communication, we show that the ground state has only CDW insulators, and more importantly, the SS phase occupies a much larger region in the phase diagram, existing up to very large hopping values which could be orders of magnitude higher than that of the well-known case. In particular, the SS-superfluid phase boundary increases linearly as a function of hopping when $2 d V \gtrsim 1.5 U$, for which the prospects of observing the SS phase with dipolar Bose gases loaded into optical lattices is much higher.
\end{abstract}

DOI: 10.1103/PhysRevA.83.051606

Can a solid be superfluid? The so-called supersolid phase is characterized by the simultaneous existence of diagonal (crystalline) and off-diagonal (superfluid) long-range orders. Although this intriguing possibility was suggested a long time ago in the context of solid ${ }^{4} \mathrm{He}[1]$, and in spite of numerous attempts over the past decades, convincing experimental evidence for its existence is yet to be found [2]. On the one hand, there is still some controversy in the condensed-matter literature regarding the recent reports that the theoretically predicted nonclassical rotational inertia was found in solid ${ }^{4} \mathrm{He}$ with the torsional oscillator experiments [3]. On the other hand, there is strong theoretical evidence that the situation in lattice models is promising [4,5], which could be advanced with ultracold quantum gases loaded into optical lattices [6].

Possibly the simplest models that show SS behavior are the extended-type Bose-Hubbard ones with on-site and nearest-neighbor (NN) repulsions. Since these models can be naturally realized with dipolar bosons [7], i.e., bosonic atoms or molecules with permanent or induced magnetic or electric dipole moments, the ground-state phase diagrams of various extended models have already been studied. For instance, the existence and stability of SS phases have been demonstrated via the Gutzwiller ansatz [4,8,9] and decoupling mean-field [10] approaches, and numerically exact quantum Monte Carlo [5,11] techniques. The SS phase is known to be very fragile, and one of the major obstacles in creating and observing it is its very small existence region and low critical temperatures. In a very recent proposal [12], it has been suggested that one way of overcoming these difficulties is to load high occupancies of bosons into optical lattices. Such systems are well described by the quantum rotor model, for which the mean-field calculation gives linear dependence between the critical hopping $\left(t_{c}\right)$ and occupancy.

In this Rapid Communication, we use the Gutzwiller ansatz and mean-field decoupling to analyze the phase diagram of the extended Bose-Hubbard Hamiltonian with on-site $(U)$ and isotropic $\mathrm{NN}(V)$ repulsions. For $d$-dimensional hypercubic lattices, beyond the critical threshold $2 d V>U$, we show that the SS phase occupies a much larger region in the phase diagram, existing up to very large hopping values of the order of $2 d t_{c} \gtrsim U$. In particular, the SS-superfluid phase boundary increases linearly as a function of hopping when $2 d \mathrm{~V} \gtrsim 1.5 \mathrm{U}$. This must be contrasted with the below-threshold $(2 d V<U)$
PACS number(s): 03.75.-b, 67.80.kb, 67.85.Hj

case, for which it is well known that the SS phase occupies small regions around the CDW insulators, existing only up to $2 d t_{c} \lesssim 0.4 U$. Therefore, we show that the prospect of observing the SS phase is much higher when $2 d V>U$. We also argue that our results for two dimensions are directly applicable to the quasi-two-dimensional dipolar Bose gases, for which the condition $4 V>U$ could be easily achieved by tuning the $s$-wave scattering length using the Feshbach resonances.

Hamiltonian. To obtain these results, we use the extended Bose-Hubbard Hamiltonian with an isotropic NN repulsion,

$$
\begin{aligned}
H= & -t \sum_{\langle i, j\rangle}\left(b_{i}^{\dagger} b_{j}+b_{j}^{\dagger} b_{i}\right)+\frac{U}{2} \sum_{i} \widehat{n}_{i}\left(\widehat{n}_{i}-1\right) \\
& +V \sum_{\langle i, j\rangle} \widehat{n}_{i} \widehat{n}_{j}-\mu \sum_{i} \widehat{n}_{i},
\end{aligned}
$$

where $t$ is the tunneling (or hopping) amplitude between NN sites $i$ and $j, b_{i}^{\dagger}\left(b_{i}\right)$ is the boson creation (annihilation) operator at site $i, \widehat{n}_{i}=b_{i}^{\dagger} b_{i}$ is the boson number operator, and $\mu$ is the chemical potential. As it turns out, the phase diagram of this Hamiltonian depends strongly on the relative strength $U-z V$, where $z=2 d$ is the lattice coordination number, leading to the following two important cases.

(i) Weak NN coupling $(z V<U)$ : When $V \neq 0$, the ground state has two types of insulating phases $[8,15]$. The first one is the Mott insulator (MI) where, similar to the usual BoseHubbard model, the average boson occupancy is the same for every lattice site, i.e., $\left\langle\widehat{n}_{i}\right\rangle=n_{0}$. Here, $\langle\cdots\rangle$ is the thermal average, and $n_{0}$ is chosen to minimize the ground-state energy for a given $\mu$. The second one is the CDW insulator, which has crystalline order in the form of staggered average occupancies. To describe the CDW insulator, it is convenient to split the entire lattice into two sublattices (e.g., $A$ and $B$ ) such that the NN sites belong to a different sublattice, i.e., $\left\langle\widehat{n}_{i}\right\rangle=n_{A}$ and $\left\langle\widehat{n}_{j}\right\rangle=n_{B}$ for $\langle i, j\rangle$. We assume the occupancies are such that $n_{A} \geqslant n_{B}$, and the case with $n_{A}=n_{B}$ corresponds to the Mott insulator.

In the atomic $(t=0)$ limit, it turns out that the chemicalpotential width of all CDW and Mott insulators is $z V$ and $U$, respectively, and the ground state alternates between the CDW and Mott phases as a function of increasing $\mu$. For instance, the ground state is a $\left(n_{A}=0, n_{B}=0\right)$ vacuum for $\mu \leqslant 0$; a 
(a) $z V=0.9 U$

(c) $z V=1.25 \mathrm{U}$

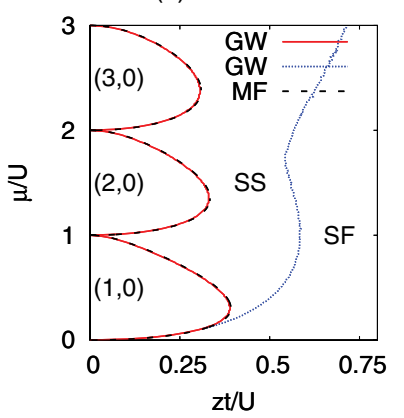

(e) $z V=2 U$

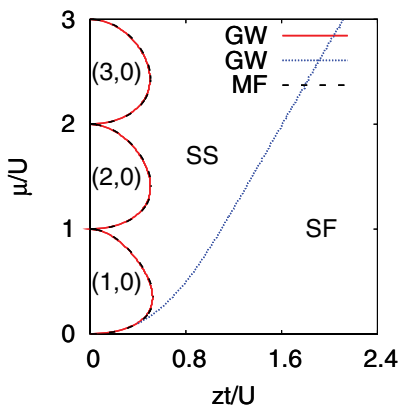

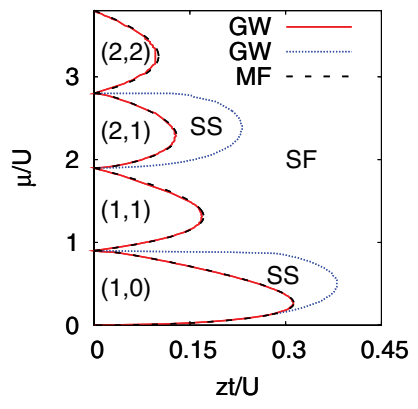

(b) $z V=1.1 \mathrm{U}$

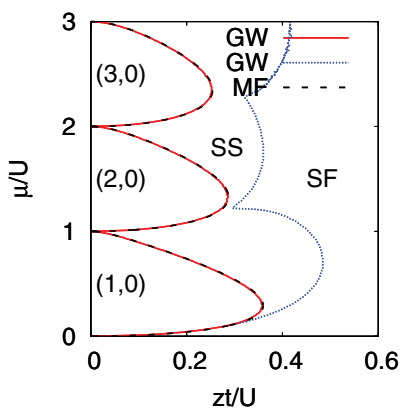

(d) $z V=1.5 \mathrm{U}$

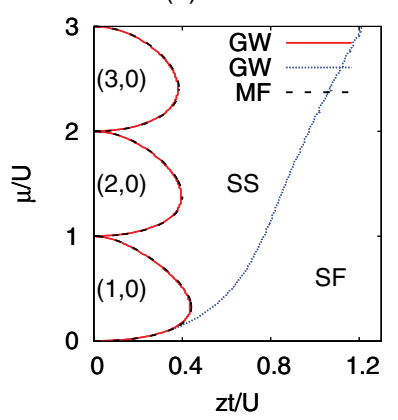

(f) $\mathrm{ZV}=4 \mathrm{U}$

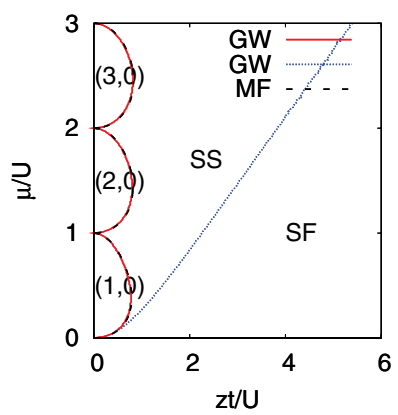

FIG. 1. (Color online) The ground-state phase diagrams are shown as a function of the chemical potential $\mu$ and hopping $t$ for $z V=$ (a) $0.9 U$, (b) $1.1 U$, (c) $1.25 U$, (d) $1.5 U$, (e) $2 U$, and (f) $4 U$. The CDW and Mott insulators are indicated with their sublattice occupancies $\left(n_{A}, n_{B}\right)$. Here, the red solid and blue dotted lines are obtained from the Gutzwiller ansatz calculations, and the black dashed lines are obtained from Eq. (7).

$(1,0) \mathrm{CDW}$ insulator for $0<\mu<z V$; a $(1,1)$ Mott insulator for $z V<\mu<U+z V$; a $(2,1)$ CDW insulator for $U+z V<$ $\mu<U+2 z V$; a $(2,2)$ Mott insulator for $U+2 z V<\mu<$ $2 U+2 z V$, and so on. As $t$ increases, the range of $\mu$ about which the ground state is insulating decreases, and the CDW and Mott insulators disappear at a critical value of $t$, beyond which the system becomes compressible [superfluid (SF) or SS], as shown in Fig. 1(a).

(ii) Strong $\mathrm{NN}$ coupling $(z V>U)$ : In contrast to the well-known weak NN coupling, the strong NN coupling of this model has not been studied much in the literature, which is the main topic of this Rapid Communication. When $z \mathrm{~V}$ is exactly equal to $U$, it is easy to check, at least in the atomic limit, that the $\left(n_{0}+1, n_{0}\right) \mathrm{CDW}$ insulator becomes degenerate in energy with the $\left(2 n_{0}+1,0\right) \mathrm{CDW}$ insulator, and the $\left(n_{0}, n_{0}\right)$ Mott insulator becomes degenerate with the $\left(2 n_{0}, 0\right)$
CDW insulator. This indicates that beyond the critical $z V=U$ threshold, both the CDW and Mott insulators that are found in the weak NN coupling are unstable against formation of new CDW insulators, leading to a phase diagram which has a very different qualitative structure.

In this Rapid Communication, we show that the ground state has only CDW-type insulating phases in the strong NN coupling; the chemical-potential width of all is $U$ in the atomic limit. For instance, the ground state is a $(0,0)$ vacuum for $\mu \leqslant 0$, a $(1,0) \mathrm{CDW}$ insulator for $0<\mu<U$, a $(2,0) \mathrm{CDW}$ insulator for $U<\mu<2 U$, a $(3,0) \mathrm{CDW}$ insulator for $2 U<$ $\mu<3 U$, and so on. As $t$ increases, the range of $\mu$ about which the ground state is insulating decreases, and the CDW insulators disappear at a critical hopping, beyond which the system becomes a SS, as shown in Figs. 1(b)-1(f). Most importantly, unlike the weak NN coupling where the SS phase occupies small regions in the phase diagram around the $\mathrm{CDW}$ insulators, we show that the SS phase occupies a much larger region in this case, existing up to very large hopping values, which could be orders of magnitude higher than that of the weak NN coupling. To obtain these results, we solve the Schrödinger equation for the Gutzwiller ansatz, as discussed next.

Gutzwiller ansatz. This ansatz has been frequently used to approximate the many-body wave functions of Bose-Hubbard Hamiltonians $[4,8,9]$. It can be written as

$$
|\psi\rangle=\prod_{i}\left(\sum_{m} f_{i, m}|i, m\rangle\right),
$$

where $|i, m\rangle$ represents the Fock state of $m$ bosons occupying the site $i$, and $f_{i, m}$ is the probability amplitude of its occupation. Here, $m=0,1, \ldots, m_{\max }$, where $m_{\max }$ is the maximum number of bosons allowed in the numerics in which we typically choose $m_{\max }=50$. The normalization of the wave function $\langle\psi \mid \psi\rangle$ requires $\sum_{m}\left|f_{i, m}\right|^{2}=1$ for each $i$.

Within this ansatz, the superfluid order parameter $\phi_{i}=$ $\left\langle\psi\left|b_{i}\right| \psi\right\rangle$ is determined by

$$
\phi_{i}=\sum_{m} \sqrt{m+1} f_{i, m}^{*} f_{i, m+1} .
$$

This complex parameter describes the state of the system at site $i$ : while it vanishes for the CDW and Mott insulators, it is finite for the SF and SS ground states. Therefore, $\phi_{i} \rightarrow 0^{+}$signals the phase boundary between an insulating and a compressible phase. Similarly, the average occupancy $n_{i}=\left\langle\psi\left|b_{i}^{\dagger} b_{i}\right| \psi\right\rangle$ is determined by

$$
n_{i}=\sum_{m} m\left|f_{i, m}\right|^{2} .
$$

In Eqs. (3) and (4), the probability amplitudes are obtained by solving the Schrödinger equation, $\langle\psi|H| \psi\rangle=$ $i \hbar\langle\psi|\partial| \psi\rangle / \partial \tau$, with $f_{i, m}=f_{0, i, m} e^{-i \epsilon_{0, i} \tau / \hbar}$. This leads to

$$
\begin{aligned}
\epsilon_{0, i} f_{0, i, m}= & -t\left(\bar{\phi}_{i} \sqrt{m} f_{0, i, m-1}+\bar{\phi}_{i}^{*} \sqrt{m+1} f_{0, i, m+1}\right) \\
& +\left[\frac{U}{2} m(m-1)+V m \bar{n}_{i}-\mu m\right] f_{0, i, m},
\end{aligned}
$$

where $\bar{\phi}_{i}=\sum_{\langle j\rangle_{i}} \phi_{j}$ and $\bar{n}_{i}=\sum_{\langle j\rangle_{i}} n_{j}$ sum over sites $j$ neighboring to site $i$.

For the ground state, first we need the minimal eigenvalue $\epsilon_{0, i}$ and the elements $f_{0, i, m}$ of the corresponding eigenvector, 
and we then use them in Eqs. (3) and (4) to solve for $\phi_{i}$ and $n_{i}$ self-consistently. Note that in uniform systems with two sublattices discussed in this Rapid Communication, the state of the whole system is sufficiently described by the two order parameters $\phi_{A}$ and $\phi_{B}$ for the sublattices $A$ and $B$, respectively. When this is the case, note also that $\epsilon_{0, i}=\epsilon_{0, A}$, $f_{0, i, m}=f_{0, A, m}, \bar{\phi}_{i}=z \phi_{B}, n_{i}=n_{A}$, and $\bar{n}_{i}=z n_{B}$ for $i \in A$ sublattice, and $\epsilon_{0, i}=\epsilon_{0, B}, f_{0, i, m}=f_{0, B, m}, \bar{\phi}_{i}=z \phi_{A}, n_{i}=n_{B}$, and $\bar{n}_{i}=z n_{A}$ for $i \in B$ sublattice. To support our Gutzwiller ansatz calculations, next we examine the mean-field theory, which provides an analytical expression for the phase boundary between the insulating and compressible phases.

Mean-field decoupling approximation. In constructing the mean-field theory, one first defines the SF order parameter $\phi_{i}=\left\langle b_{i}\right\rangle$, and then replaces the operator $b_{i}$ with $\phi_{i}+\delta b_{i}$ in the hopping terms of Eq. (1). This approximation decouples the two-particle hopping terms into single-particle ones, and the resultant mean-field Hamiltonian can be solved via exact diagonalization in a power series of $\phi_{i}$. Performing a secondorder perturbation theory in $\phi_{i}$ around the insulators, and following the usual Landau procedure for second-order phase transitions, i.e., minimizing the energy as a function of $\phi_{i}$, we eventually arrive at the condition

$\phi_{i}=\bar{\phi}_{i} t\left[\frac{n_{i}+1}{U n_{i}+V \bar{n}_{i}-\mu}-\frac{n_{i}}{U\left(n_{i}-1\right)+V \bar{n}_{i}-\mu}\right]$,

where the definitions of $\bar{\phi}_{i}$ and $\bar{n}_{i}$ are the same as in Eq. (5). It is known that the results of the mean-field theory coincide with those of the Gutzwiller ansatz, and that they both become exact when $d \gg 1$ [13]. We emphasize that the mean-field calculations give a good qualitative description of the system, and it becomes progressively accurate as the dimensionality and/or the occupancy increases.

For uniform systems with two sublattices, Eq. (6) gives coupled equations for $\phi_{A}$ and $\phi_{B}$, which can be solved to obtain the phase boundary between the insulating (CDW or Mott) and compressible (SF or SS) phases. Since $\phi_{A}, \phi_{B} \rightarrow 0^{+}$ near these boundaries, Eq. (6) can be satisfied only if [14]

$$
\begin{aligned}
\frac{1}{z^{2} t^{2}}= & {\left[\frac{n_{A}+1}{U n_{A}+z V n_{B}-\mu}-\frac{n_{A}}{U\left(n_{A}-1\right)+z V n_{B}-\mu}\right] } \\
& \times\left[\frac{n_{B}+1}{U n_{B}+z V n_{A}-\mu}-\frac{n_{B}}{U\left(n_{B}-1\right)+z V n_{A}-\mu}\right],
\end{aligned}
$$

which gives a quartic equation for $\mu$. An alternative way of deriving this equation is the random-phase approximation [15]. Since a simple closed-form analytic solution for $\mu$ is not possible, we solve Eq. (7) for each of the insulating lobes separately. Having discussed the details of the Gutzwiller ansatz and mean-field approximation, we are ready to discuss the phase diagrams.

Phase diagrams. We solve Eqs. (3)-(5) self-consistently for the order parameters (i.e., $\phi_{A}$ and $\phi_{B}$ ) and average occupancies (i.e., $n_{A}$ and $n_{B}$ ), and use them to construct the phase diagram of the system. The CDW and Mott insulators are characterized by $\phi_{A}=\phi_{B}=0$, and $n_{A} \neq n_{B}$ and $n_{A}=n_{B}$, respectively. However, the SF and SS phases are characterized by $\phi_{A}=$ $\phi_{B} \neq 0$ and $\phi_{A} \neq \phi_{B}$, respectively, which naturally leads to $n_{A}=n_{B}$ in the SF phase and $n_{A} \neq n_{B}$ in the SS phase. In (a) $\phi_{A}-\phi_{B}$

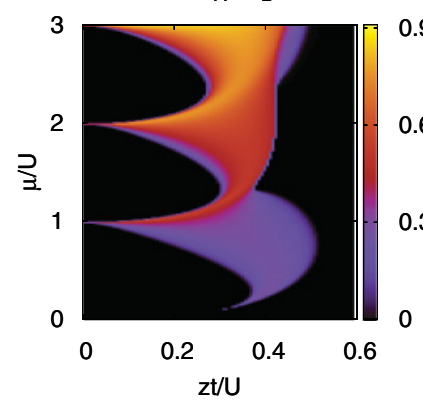

(b) $n_{A}-n_{B}$

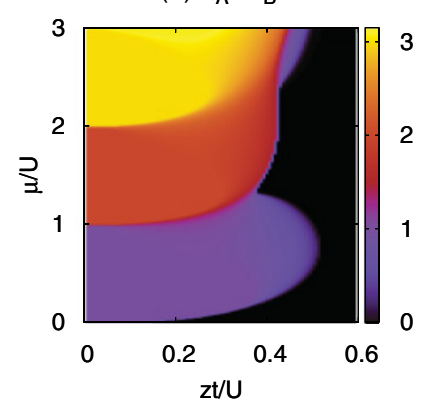

FIG. 2. (Color online) The colored maps of the (a) relative order parameter $\phi_{A}-\phi_{B}$ and (b) relative average occupancy $n_{A}-n_{B}$ are shown as a function of the chemical potential $\mu$ and hopping $t$ when $z V=1.15 U$.

this Rapid Communication, we choose $\phi_{A}$ and $\phi_{B}$ to be real, since we are only interested in the CDW-SS and MI-SF phase boundaries which are determined by $\left\{\phi_{A}, \phi_{B}\right\} \neq 0$, and the SSSF phase boundaries which are determined by $\phi_{A}=\phi_{B} \neq 0$.

Therefore, it is sufficient to look at the relative order parameter $\phi_{A}-\phi_{B}$ and relative average occupancy $n_{A}-n_{B}$ to distinguish between various phases. For instance, in Fig. 2, we show the colored maps of $\phi_{A}-\phi_{B}$ and $n_{A}-n_{B}$ as a function of the chemical potential $\mu$ and hopping $t$ for $z V=1.15 U$. The three dark lobes shown in Fig. 2(a), where $\phi_{A}=\phi_{B}=0$, correspond to $(1,0),(2,0)$, and $(3,0) \mathrm{CDW}$ insulators (from bottom to top), the occupancies of which are clearly seen in Fig. 2(b). Here, the SF phase occupies the dark region that is common in both Figs. 2(a) and 2(b). It is also clear that the SS phase, where $\phi_{A}-\phi_{B} \neq 0$ and $n_{A}-n_{B} \neq 0$, shown with bright colors in Figs. 2(a) and 2(b), respectively, is sandwiched between the CDW insulators from the left and SF phase from the right. Note that, in the SS phase, the superfluid order parameter is larger on the sublattice with higher occupancy, and both the crystalline and superfluid orders are primarily on the same sublattice [16]. This is because the particle and hole excitation energies are higher on the sublattice with lower occupancy when $z V>U$.

In Fig. 1, we repeat this analysis for a number of $\mathrm{NN}$ repulsions, and plot the phase diagrams as a function of $\mu$ and $t$. In these figures, the red solid lines and blue dotted lines are obtained from the Gutzwiller ansatz calculations, and the black dashed lines are obtained from Eq. (7). Note that both methods are in complete agreement, i.e., on top of each other for the phase boundary between the insulating (CDW or Mott) and compressible (SF or SS) phases, which supports our Gutzwiller ansatz calculations.

A typical weak-NN-coupling phase diagram is shown in Fig. 1(a) for $z V=0.9 U$. As discussed above, the ground state alternates between the CDW and Mott insulators as a function of increasing $\mu$, and the SS phase occupies only small regions around the CDW insulators. Unlike the weak-NN-coupling phase diagrams, Figs. 1(b)-1(f) show that the ground state has only CDW-type insulating phases in the strong NN coupling. In particular, the chemical-potential width of all CDW insulators is $U$ in the atomic limit, and the ground state is a $\left(n_{0}, 0\right) \mathrm{CDW}$ insulator for $\left(n_{0}-1\right) U<\mu<n_{0} U$. As $t$ increases, the range of $\mu$ about which the ground state is insulating decreases, 
and the CDW insulators disappear at a critical value of $t_{c}$, beyond which the system becomes a SS. Note that, except for the weak-NN-coupling phase diagram, all of the $t_{c}$ values are comparable to each other for a fixed $z V$.

The main result of this Rapid Communication is shown in Figs. 1(c)-1(f). It is clearly seen that the SS phase occupies a much larger region when $z V \gtrsim 1.1 U$, existing up to very large hopping values, which could be orders of magnitude higher than that of the weak NN coupling. This result is intuitive given that the ground state has only $\left(n_{0}, 0\right) \mathrm{CDW}$ insulators whose sizes are comparable to each other, and the CDW modulations become stronger as $n_{0}$ (or $\mu$ ) increases. In fact, the SS-SF phase boundary becomes linear in $\mu$ and $z t$ when $z V \gtrsim 1.5 U$. Our numerical calculations suggest that the slope of this line is approximately given by $d \mu / d(z t)=2 /(z V / U-1)$. A similar linear dependence between the particle density and hopping has recently been found for the SS-SF phase-transition boundary in the case of the quantum rotor model [12], when the average occupation is much higher than unity.

Experimental realization. Here, we argue that our results for two dimensions $(z=4)$ is directly applicable to the quasi-two-dimensional dipolar Bose gases. For the optical lattice potential $V_{\mathrm{OL}}(r)=V_{0}\left[\sin ^{2}(k x)+\sin ^{2}(k y)\right]$, where $k=2 \pi / \lambda$ is the wave vector and $\ell=\lambda / 2$ is the lattice spacing, the on-site interaction depends on the $s$-wave scattering length $a_{s}$ via [6] $U=\sqrt{8 / \pi} k a_{s} E_{r} s^{3 / 4}$. Typically, $s=V_{0} / E_{r} \sim 10$, where $E_{r}=\hbar^{2} k^{2} /(2 m)$ is the recoil energy and $m$ is the particle mass. Assuming all of the dipoles are polarized along the $z$ direction, the dipole-dipole interaction becomes isotropic, leading to $V=C_{\mathrm{dd}} /\left(4 \pi \ell^{3}\right)$ for the NN repulsion, where $C_{\mathrm{dd}}=\mu_{0} p^{2}$ (or $p^{2} / \varepsilon_{0}$ ) for particles with permanent magnetic (or electric) dipole moment $p$. The ratio $U /(4 V)=\pi^{2} \sqrt{2 \pi} s^{3 / 4} /\left(12 \varepsilon_{\mathrm{dd}}\right)$, where $\varepsilon_{\mathrm{dd}}=a_{\mathrm{dd}} / a_{s}$ and $a_{\mathrm{dd}}=m C_{\mathrm{dd}} /\left(12 \pi \hbar^{2}\right)$ is the dipolar length scale, determines the critical threshold $(4 V>U)$. For instance, $p=6 \mu_{B}$ and $a_{\mathrm{dd}}=16 a_{0}$ for the ${ }^{52} \mathrm{Cr}$ atoms [17], where $\mu_{B}\left(a_{0}\right)$ is the
Bohr magneton (radius), and the condition is $a_{s} \lesssim 8 a_{0} / s^{3 / 4}$. However, $p=0.6$ Debye and $a_{\mathrm{dd}}=2 \times 10^{3} a_{0}$ for the $\mathrm{KRb}$ molecules [17], and the condition is $a_{s} \lesssim 10^{3} a_{0} / \mathrm{s}^{3 / 4}$. Since $a_{s} \sim 100 a_{0}$ for Cr atoms, $a_{s}$ needs to be tuned via a Feshbach resonance [18] in order to achieve the critical threshold.

In addition, we note that it is easy to extract the finite-size effects of an external trapping potential (e.g., present in atomic systems) from Fig. 1. For instance, within the local-density approximation, if the center of the trap is a $(2,0) \mathrm{CDW}$ insulator (say $z t \lesssim 0.6 U$ ), the system is expected to first go through a SS and then a $(1,0) \mathrm{CDW}$ insulator before becoming a SF as a function of the radial distance toward the edge of the trap. However, if the center of the trap is a SS (say $z t \gtrsim 0.6 U$ ), the system is expected to become a SF beyond a critical radius, without any intermediate phase.

Conclusions. To summarize, beyond the critical threshold $z V>U$, we showed that the SS phase occupies a much larger region in the phase diagram, existing up to very large hopping values of the order $z t \gtrsim U$, and that the SS-SF phase boundary increases linearly as a function of $t$ when $z V \gtrsim 1.5 U$. Therefore, our results suggest that the prospect of observing the SS phase is much higher when $z V>U$, which could be easily achieved with quasi-twodimensional dipolar Bose gases loaded into optical lattices by tuning the $s$-wave scattering length via currently available Feshbach techniques [18]. We believe our mean-field treatment captures the qualitative physics correctly, and that this work will motivate further quantum Monte Carlo calculations in the strong-NN-coupling regime for more-accurate phase diagrams.

Acknowledgments. The author thanks J. K. Freericks for comments. This work is financially supported by the Marie Curie International Reintegration (Grant No. FP7PEOPLEIRG-2010-268239) and the Scientific and Technological Research Council of Turkey (Career Grant No. TÜBİTAK3501-110T839).
[1] A. F. Andreev and I. M. Lifshitz, Sov. Phys. JETP 29, 1107 (1969); G. V. Chester, Phys. Rev. A 2, 256 (1970); A. J. Leggett, Phys. Rev. Lett. 25, 1543 (1970).

[2] A. J. Leggett, Science 305, 1921 (2004).

[3] E. Kim and M. H. W. Chan, Nature (London) 427, 225 (2004); Science 305, 1941 (2004); S. Sasaki et al., ibid. 313, 1098 (2006); J. D. Reppy, Phys. Rev. Lett. 104, 255301 (2010).

[4] K. Góral, L. Santos, and M. Lewenstein, Phys. Rev. Lett. 88, 170406 (2002).

[5] P. Sengupta, L. P. Pryadko, F. Alet, M. Troyer, and G. Schmid, Phys. Rev. Lett. 94, 207202 (2005).

[6] I. Bloch, J. Dalibard, and W. Zwerger, Rev. Mod. Phys. 80, 885 (2008).

[7] J. Stuhler et al., Phys. Rev. Lett. 95, 150406 (2005); T. Koch et al., Nature Phys. 4, 218 (2008); B. Pasquiou et al., Phys. Rev. Lett. 106, 015301 (2011).

[8] D. L. Kovrizhin, G. Venketeswara Pai, and S. Sinha, Europhys. Lett. 72, 162 (2005).

[9] C. Menotti, C. Trefzger, and M. Lewenstein, Phys. Rev. Lett. 98, 235301 (2007).
[10] S. Yi, T. Li, and C. P. Sun, Phys. Rev. Lett. 98, 260405 (2007); I. Danshita and C. A. R. Sá de Melo, ibid. 103, 225301 (2009).

[11] A. van Otterlo and K.-H. Wagenblast, Phys. Rev. Lett. 72, 3598 (1994); G. G. Batrouni, R. T. Scalettar, G. T. Zimanyi, and A. P. Kampf, ibid. 74, 2527 (1995); B. Capogrosso-Sansone, C. Trefzger, M. Lewenstein, P. Zoller, and G. Pupillo, ibid. 104, 125301 (2010); L. Pollet, J. D. Picon, H. P. Buchler, and M. Troyer, ibid. 104, 125302 (2010).

[12] A. Bühler and H. P. Büchler, e-print arXiv:1012.3341.

[13] D. S. Rokhsar and B. G. Kotliar, Phys. Rev. B 44, 10328 (1991).

[14] M. Iskin and J. K. Freericks, Phys. Rev. A 79, 053634 (2009).

[15] M. Iskin and J. K. Freericks, Phys. Rev. A 80, 063610 (2009).

[16] This is in contrast to the weak NN coupling, where the particle and hole excitation energies are higher on the sublattice with higher occupancy, i.e., the $A$ sublattice, leading to a crystalline (superfluid) order primarily on the sublattice $A(B)$.

[17] See T. Lahaye et al., Rep. Prog. Phys. 72, 126401 (2009) for a recent review of experiments with dipolar Bose gases.

[18] T. Lahaye et al., Nature (London) 448, 672 (2007). 